\title{
Study of the sealing elements impact on air flow distribution in a seed vessel of seeding mechanism
}

\author{
Anton Yu. Popov ${ }^{1, *}$, and Denis K. Muratov ${ }^{1}$ \\ ${ }^{1}$ Don State Technical University, 1 Gagarina sq., Rostov-on-Don, Russia, 344000
}

\begin{abstract}
The quality of the pneumatic seeding mechanism operation is defined by a variety of factors, including aerodynamic characteristic quantities. But aerodynamics of seeding systems is poorly studied. Therefore studying of the air flow distribution in a seeding mechanism seed vessel is considered to be a current issue. Overpressure seeding mechanism with a seed vessel compulsory sealing is approved as the object of the studying. The aim of the studying is to define the impact of the sealing elements shape on the air flow distribution in a seed vessel by various seeding mechanism operating modes. The building-up of the air flow distribution model is carried out at the software environment Solidworks Flow Simulation with allowance for a seeding wheel rotation. The aerodynamic model building-up technique is given. The graphic charts of airflow velocity change in an upper part of the seed vessel are made. The designed overpressure seeding mechanism aerodynamic model is analyzed.
\end{abstract}

\section{Introduction}

At present seeding machines with pneumatic seeding mechanisms are the most widespread type of machines to seed cultivated crop. A number of factors affect the performance quality. For instance, the pressure in a seed vessel, the seeding wheel rotation, the extra seed dropper nozzle diameter etc. Conditions, which refer to aerodynamic characteristic quantities, contribute significantly to the seed batching quality of the seeding mechanism. Thus, it's well-known, that the amount of vacuum and pressure influence seed scorching and holding by the seeding wheel batching element, and extra seed dropping is carried out by the air flow of the pneumatic dropper etc. [1-4]

But, aerodynamics of pneumatic seeding mechanisms and, particularly, the air flow distribution, its velocities and pressure, are insufficiently explored. The majority of aerodynamic processes researches add up either to analytic studying of batching processes, based on the mathematic models building-up, or to empiric studying, defining the seed batching measure reliance on various factors, including aerodynamic ones $[4,5]$. But generally air flow aerodynamic characteristic quantities and seeding system technological characteristic quantities are implicit. Besides, the most scientific studies do not disclose the

\footnotetext{
* Corresponding author: popov a ju@mail.ru
} 
full picture of the seed batching process, especially as regards the air flow distribution and the effects of structural elements inside the seeding mechanism pneumatic seed vessel. That's why, studying and modeling of the air flow distribution processes within the seed vessel and the determination of the influence of the seeding mechanism structural elements are an urgent task.

\section{Design and function of the pneumatic seeding mechanism}

Vacuum or pressure can be applied for seed batching in seeding mechanisms. According to it, pneumatic seeding mechanisms can be divided into vacuum and overpressure mechanisms $[1,2,5]$.

As an object of our study we'll view the seeding mechanism, working at overpressure [5] and presented at the fig. 1a. This very type of pneumatic mechanisms, despite the vacuum ones, provides high seed batching measures at high rate of sowing.

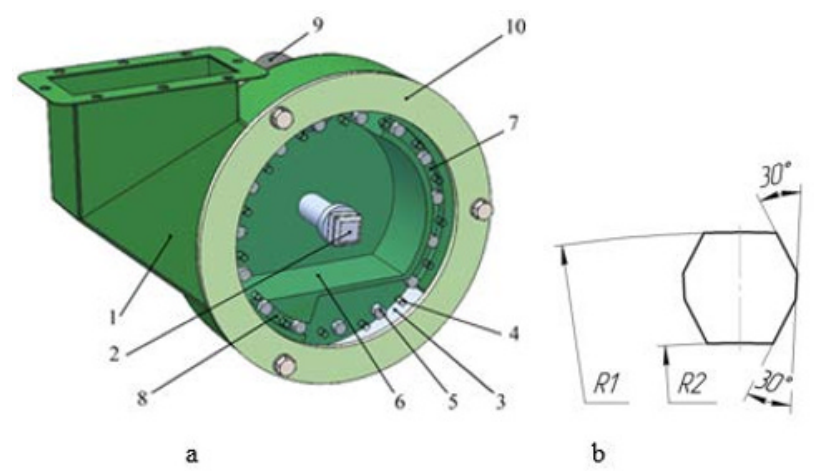

Fig. 1. General view of the overpressure seeding mechanism (a) and dimensions of the protuberance (b): R1 and R2 - the radii of the curvilinear walls; 1 - housing; 2 - shaft; 3 - seeding disk; 4 - aperture of the metering element; 5 - protuberance; 6 - transport channel; 8 - input channel; 9 - nozzle; 10 cover.

The overpressure pneumatic mechanism operates the following way.

Seeds are filled from the hopper by gravity from the seed vessel. The air is injected into the seed vessel through the nozzle to discard excess seeds and creates an overpressure. Seeds under the influence of overpressure are captured by the batching elements of the rotating seeding wheel. Seized seeds are transferred to the upper part of the seed vessel, where excess seeds are removed by the air flow created by the nozzle and then into the transport channel. Being in the transport channel, the batching element with the seed is cut off by the projection from the effect of overpressure. The protuberances have a complex shape, which is a curvilinear quadrilateral with cut corners (Fig. 1b). Some sides of the protuberances are rectilinear, while others are bounded by arcs concentric to the axis of seeding wheel rotation. Performing protuberances with cut off angles of $30^{\circ}$ will ensure a guaranteed ejection of seeds without pinching [6]. In the transport channel, the seeds move to the lower part of the mechanism body, where they are dumped into the opener.

A distinctive feature of the seeding mechanism under consideration from other overpressure devices is the use of protuberances and a transport channel made in the mechanism body to isolate the seed vessel from the external environment during batching elements capture, transportation and discharge of seeds into the opener. However, the protuberances, along with merits, as sealing elements, have a disadvantage. They worsen the quality of dumping excess seeds in the upper part of the seed vessel, that is, they increase two-piece, three-piece feeds and feedings of higher order. This can be seen from 
empiric studies. So increasing the distance between the protuberances from 22 to $28 \mathrm{~mm}$ leads to a change in the frequency of two-piece feeds of corn seeds in the range from $2.6 \%$ to $3.6 \%$. [5]. In addition, from previous studies, it follows that the shape of the protuberances affects the batching of the seeds, in particular the damage to the seeds and the quality of their supply [6].

\section{Aim and tasks of the study}

The air flow velocity of the seeding wheel spreading in the longitudinal plane must be in the range of the speed of waving or higher to ensure that extra seed are thrown off by air. It is known from hydrodynamics that the shape of a physical body influences the character of the air flow distribution. Consequently, the shape of the outriggers of the seeding wheel influences the air flow distribution in the discharge zone of the extra seeds of the seeding mechanism.

But because of the complexity of the aerodynamic processes and the large number of factors of a constructive and technological nature occurring in the enclosed space of the seeding mechanism, the air distribution is ambiguous and highly dependent on the structural features of the seed vessel and operating modes, such as the air flow entering the seed vessel, seeding wheel rotation etc.

Therefore, the aim of the study is to determine the effect of the sealing elements shape on the air flow distribution in the seed vessel in the overpressure mechanism under consideration.

To achieve the aim of the study, it is necessary to solve the following tasks: 1) to develop a model for constructing the aerodynamic model of the mechanism; 2) to determine the initial and boundary conditions of the model and the criteria for the convergence of the calculation; 3) to simulate and analyze the air flow velocity distribution in the seeding mechanism seed vessel, in particular in the zone of dropping extra seeds at different air flow rates.

\section{Methods of the study}

With the development of software and hardware base of computer technology and the possibility of carrying out numerical spatial modeling of air flows, it became possible to use computer simulation on the basis of numerical calculations as an a priori method of aerodynamic processes studying occurring in the seeding mechanism [7]. For computer simulation of air flow distribution, the Solidworks Flow Simulation software module is well suited for solving gas dynamics applications. In Solidworks Flow Simulation, fluid motion is calculated on the basis of the Navier-Stokes equations [8].

The algorithm for constructing the mechanism aerodynamic model consists of the following stages: 1) development of a pneumatic mechanism spatial model for precise seeding; 2) determination of the calculation area; 3) definition and specification of the rotation local area; 4) setting the boundary conditions; 5) construction of the initial calculation grid and determination of its adaptation parameters by solution and boundary conditions; 6) definition and assignment of surface aims; 7) performance of calculation and estimation of accuracy by the grid convergence method; 8) graphical representation of calculation results.

For the aerodynamic calculation, the following initial conditions are defined: air is chosen as a fluid; ambient temperature $-20{ }^{\circ} \mathrm{C}$; The rest of the characteristic quantities are left by default. The following boundary conditions of the model are determined: the air flow volume at the inlet to the nozzle of the pneumatic mechanism is set at levels 15,25 
and $35 \mathrm{l} / \mathrm{s}$; the ambient pressure is set at $101325 \mathrm{~Pa}$. Levels of variation in air flow are selected on the basis of previous experimental studies and are due to the cultivated crop and seeding rate [5]. The static pressure on the surface of the seeding wheel on the seed vessel side and the air flow volume at the outlet through the holes of the seeding wheel batching elements are taken as criteria for the convergence of the calculation.

Since the seed batching process is based on the uniform rotation of the seeding wheel, this aspect must be taken into account when constructing the aerodynamic model of the seeding mechanism. To do this, the shaft and the seeding wheel with the projections are assigned a rotating area, by setting the local rotation area using the Solidworks Flow Simulation software toolkit [8]. The rotation frequency of the seeding wheel is adopted at the level of $50 \mathrm{rpm}$, which corresponds to the average rates of seed sowing for most tilled crops [5]. The entrance to the model is equipped with a nozzle for a seed dropper. Seeding wheel batching elements are taken as an output.

\section{Calculation results}

As a result of the calculation, isolines for the air flow velocity distribution in the frontal projection of the seeding wheel at different air flow rates, which are shown in Fig. 2. At the same time, the air flow velocity is recorded in the plane of the parallel front surface of the wheel with a displacement of $3 \mathrm{~mm}$, which corresponds to about half the nominal diameter of the seed.

To determine and analyze the influence of the wheel protuberances on the air flow distribution, charts are constructed of the variation in the air flow velocity v (fig. 2) in the upper part of the seed vessel at different distances from the center of the seeding wheel along arcs concentric to its axis of rotation. The position of the arcs is shown in Figure 2a. They determine the boundaries of the location of extra seeds in the batching process. The abscissas are the distances L between the protuberances along the arcs, the concentric circles of the batching elements centers (Chart 2 of Fig. 2), and the largest (Chart 1 of Fig. 2 ) and the smallest (Chart 3 of Fig. 2) radii of the circumferences of the protuberance walls.

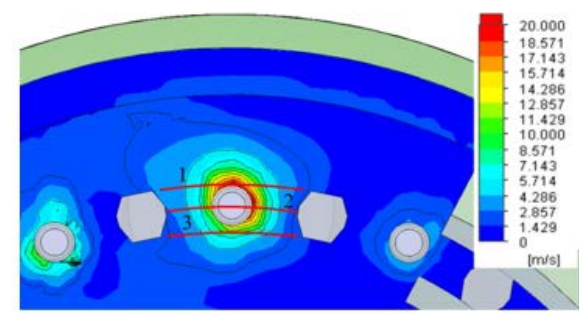

a

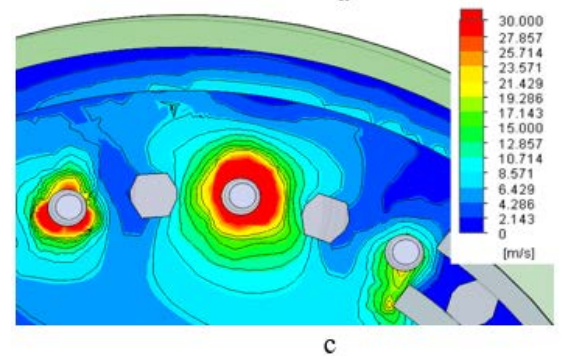

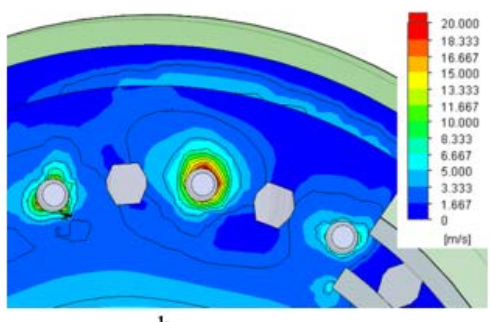

$\mathrm{b}$

Fig. 2. Air flow velocity distribution in the extra seed dropping area with air flow values of $15 \mathrm{l} / \mathrm{s}$ (a), $25 \mathrm{l} / \mathrm{s}(\mathrm{b})$ and $35 \mathrm{l} / \mathrm{s}(\mathrm{c}): 1$ - arc along the largest radius of the protuberance wall, 2 - arc along the circumference of the batching elements centers, 3 - arc along the smallest radius of the protuberance wall 


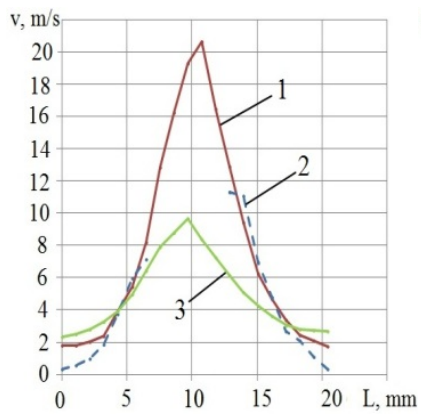

$\mathrm{a}$

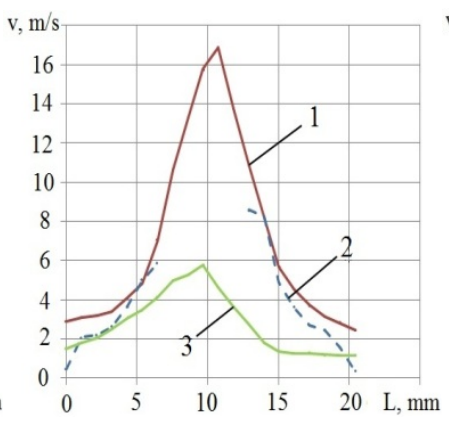

$\mathrm{b}$

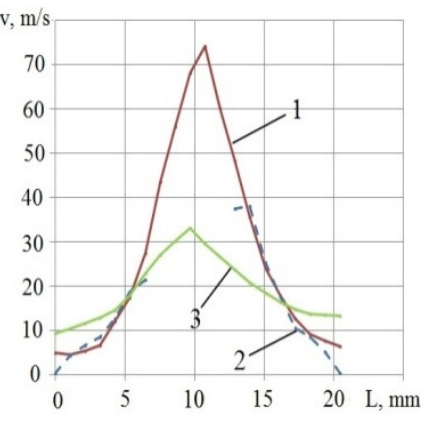

$\mathrm{c}$

Fig. 3. Change in the air flow velocity in the upper part of the seed vessel along arcs of circles between the protuberances at an air flow rate of 15 1/s (a), 25 1/s (b) and 35 1/s (c): 1 - radius of the circumferences of the protuberance walls; 2 - chart on the arc, concentric circle of the batching elements centers; 3 is a chart of the arc of the smallest radius for the protuberance wall circumferences.

On analyzing the isolines and charts of air flow distribution, the following conclusions can be drawn. At all selected levels of air flow variation, the protuberances influence the air flow distribution in the extra seed dropping area.

The greatest variation in the air flow velocity is observed at an air flow rate of $35 \mathrm{l} / \mathrm{s}$. The air flow velocity varies from 0.26 to $74.19 \mathrm{~m} / \mathrm{s}$. The smallest change in the air flow velocity is observed with an air flow rate of $25 \mathrm{l} / \mathrm{s}$ and is within the range of $0.32-16.9 \mathrm{~m} / \mathrm{s}$. At the same time, the absence of data on the section of $6-13 \mathrm{~mm}$ in terms of air flow velocity in the charts 2 of Fig. 3, is explained by the seed caught in the measurement area held at the batching element aperture.

It can be seen from the charts that in the section of $6-13 \mathrm{~mm}$ where the seed is located, at all levels of air flow rate variation, the air flow velocity is more than $4.5 \mathrm{~m} / \mathrm{s}$, which is comparable with the seed crop growth velocity of $6-15 \mathrm{~m} / \mathrm{s}$. However, at an air flow rate of $35 \mathrm{l} / \mathrm{s}$, the flow rate is much higher than the rate of seed waning and is within 24.1-74.19 $\mathrm{m} / \mathrm{s}$. This significantly increases the likelihood of removing not only extra seeds, but also the main seed held at the opening of the batching element, and can lead to an increase in zero feeds. This is confirmed by previous experimental studies of this seeding mechanism [5]. Therefore, it is recommended to set the air flow rate for this outlet design of the seeding wheel with cut corners to below $25 \mathrm{l} / \mathrm{s}$.

\section{Findings}

As a result of the study, with the help of the program module Solidworks Flow Simulation the model of air flow distribution in the overpressure seeding mechanism seed vessel was developed, taking into account the seeding wheel rotation. An algorithm for constructing an aerodynamic model is developed. The nature and analysis of the air flow distribution in the upper part of the seed vessel, in the area of dropping extra seeds in constructive design of the protuberances with cut corners is determined. The constructed model of overpressure seeding mechanism aerodynamics can be a basis for carrying out of the further researches and modernization of seeding mechanism of the given kind. 


\section{References}

1. G.Ye. Listopad, Agricultural and Meliorative Machines. (M.: Agropromizdat, 1986).

2. B. A. Stout, B. Cheze, CIGR Handbook of Agricultural Engineering, Volume III Plant Production Engineering. (American Society of Agricultural Engineers, 1999).

3. R.C.Singh, G.Singh, D.C.Saraswat, Optimisation of Design and Operational Parameters of a Pneumatic Seed Metering Device for Planting Cottonseeds, Biosystems Engineering, v. 92(4), pp. 429-438 (2005).

4. H. Z. Dizaji, M. R. Taheri, S. Minaei, Air-jet seed knockout device for pneumatic precision planters, AMA-Agricultural Mechanization in Asia Africa and Latin America, 41(1), pp. 45-50 (2010).

5. A.Yu. Popov, Investigation of Sawing Apparatus of Excessive Pressure with Forced Sealing of the Seed Chamber, Political Network Electronic Scientific Journal of KubSAU 68, p. 137-146 (2011).

6. A.Yu. Popov, Elements of Hermetic Sealing of the Sowing Device of Excessive pressure, Tractors and Agricultural Machines, No. 6, p. 32-35 (2014).

7. J.J. Yu, Y.T. Liao, J.L. Cong, S. Yang, Q.X. Liao, Simulation analysis and match experiment on negative and positive pressures of pneumatic precision metering device for rapeseed, Int J Agric \& Biol Eng, 7(3), pp. 1-12, (2014).

8. A.A. Alyamovskiy, Solid Works. Computer Modeling in Engineering Practice. (St. Petersburg: BHV- Petersburg, 2005). 